\title{
A complete design of contra-rotating propellers using the new computer system
}

\author{
Tadeusz Koronowicz, Prof. \\ Zbigniew Krzemianowski, Ph. D. \\ Teresa Tuszkowska, Ph. D. \\ The Szewalski Institute of Fluid Flow Machinery \\ of the Polish Academy of Sciences in Gdansk \\ Jan A. Szantyr, Prof. \\ Gdansk University of Technology
}

\begin{abstract}
The computer system for the complete design of the contra-rotating propellers presented in this article has several common blocks and procedures with the systems for design of single propellers and tandem corotating propellers, presented in detail in the Polish Maritime Research No.1 and No.4 of the Volume 16, 2009. In this article only the blocks and procedures developed specially for the contra-rotating propellers are described. The system is based on the lifting line and lifting surface models and on the Computational Fluid Mechanics methods. The comparative analysis of the contra-rotating propellers and the tandem corotating propellers is included.
\end{abstract}

Keywords: ship propellers, contra-rotating propellers, design methods, computational fluid dynamics

\section{INTRODUCTION}

Many years of experience and practice have shown that the classical single propellers in most cases fulfil the highest requirements regarding efficiency, cavitation characteristics, level of unsteady hydrodynamic shaft forces, pressure pulsations generated on the hull and noise/vibration. However, in realistic applications certain areas may be found in which other types of screw propellers are better . Consequently, the contra-rotating propellers (CRP) may be advantageous in some specific conditions. For example, the requirement of minimum slipstream rotation is achievable for single shaft installations only with contra-rotating propellers (a very important requirement for underwater vehicles).

The design of contra-rotating propellers is based on the same requirements and assumptions which are employed in the design of single propellers and tandem co-rotating propellers $[4,6]$. In general, the design requirements of contra-rotating are similar to those of tandem propellers, but they difer in the following characteristic features:

a) in the design program for contra-rotating propellers the determination of the aft propeller diameter is required

b) detailed information about the velocity field induced by both propellers of the set is required not only in the design program, but also in the program for analysis of the contrarotating propellers operation in the non-uniform wake.

The long-term practical experience and the analysis of the propeller-induced velocity fields shows that:

- the diameter of the aft propeller must be correlated with the diameter of the slipstream behind the forward propeller in such a way that the tip vortices shed from the forward propeller pass outside the tips of the aft propeller blades
- the number of blades of the forward and aft propellers of the contra-rotating set should be different

- the rate of rotation of both propellers of the contra-rotating set is recommended to be different.

Similarly as in the case of design of other propeller types the computer design system for contra-rotating propellers includes three interacting programs (blocks of procedures):

1) program for determination of the design velocity field for both propellers of the contra-rotating set

2) program for propeller design

3) program for analysis of the contra-rotating propellers operation in the design velocity field, including the effects of mutual interaction between both propellers.

In comparison to the system for design of single propellers the system for design of contra-rotating includes the following special elements (similarly as the system for tandem propellers):

a) procedures for the independent graphical presentation of both propellers of the set

b) procedures for determination of the design velocity field for both propellers, taking into account their mutual interaction

c) procedures for modification of the velocity field for the analysis of the contra-rotating propeller operation in the non-uniform inflow velocity field

d) procedures for determination of the induced pressure pulsations for each propeller separately and for the entire set of contra-rotating propellers

e) procedures for determination of the unsteady hydrodynamic forces and moments for each propeller separately and for the entire set of contra-rotating propellers. 


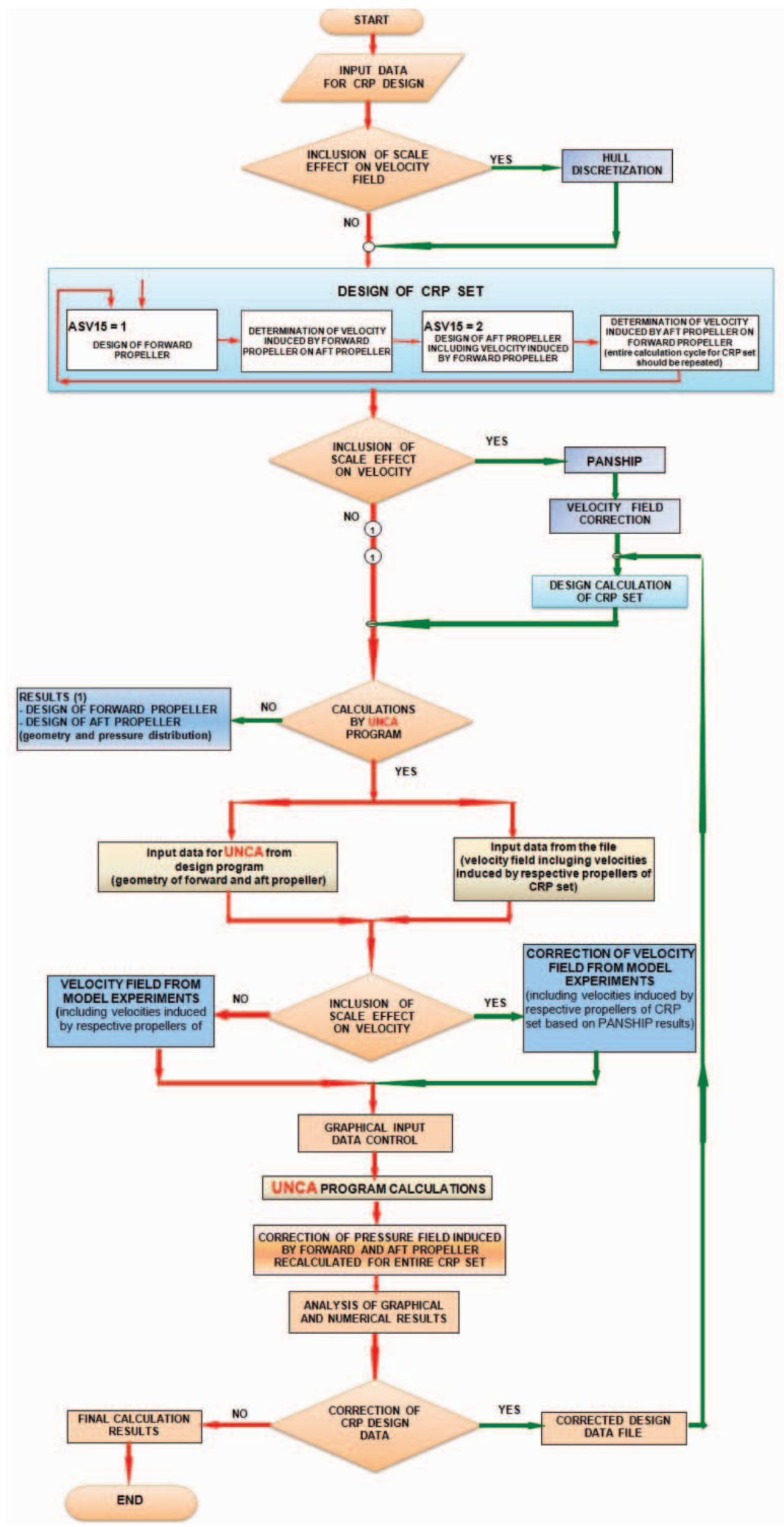

Fig. 1. The block diagram of the computer system for the complete design of the contra-rotating propellers 
The appropriate co-operation between all programs and procedures, supplemented with the above listed elements, ensures effective and complete design of the contra-rotating propellers. The system is or ganized in such a way that the designer controls the entire design process from the computer screen and the data transfer between the respective elements of the system is fully automatic.

Similarly as in the case of the previously presented systems $[4,5,6]$ an extensive use has been made of computer graphics for control and correction of the input data, for control of the intermediate results, for modification of the geometry of both propellers at consecutive stages of design and for presentation of the final results.

The block diagram of the system for design of the contrarotating propellers, shown in Fig. 1, is similar to that for design of tandem propellers [6]. The basic block diagram of the propeller design process is extensively supplemented with blocks for the design of contra-rotating propellers (cf. Fig. 2) and for the analysis of contra-rotating propellers operation in the non-uniform inflow velocity field (cf. Fig. 3).

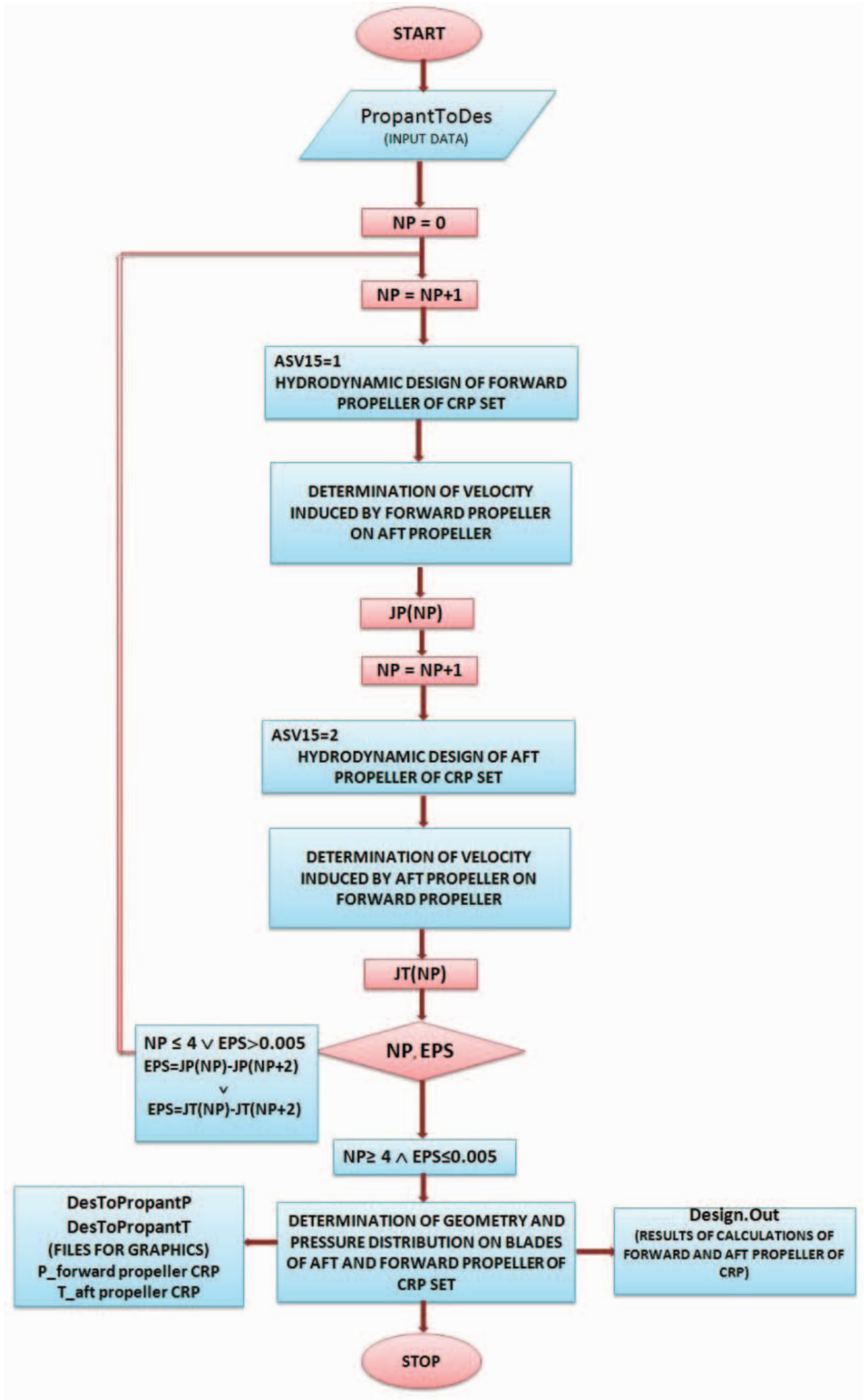

Fig. 2. The block diagram of the design procedure of the contra-rotating propellers 


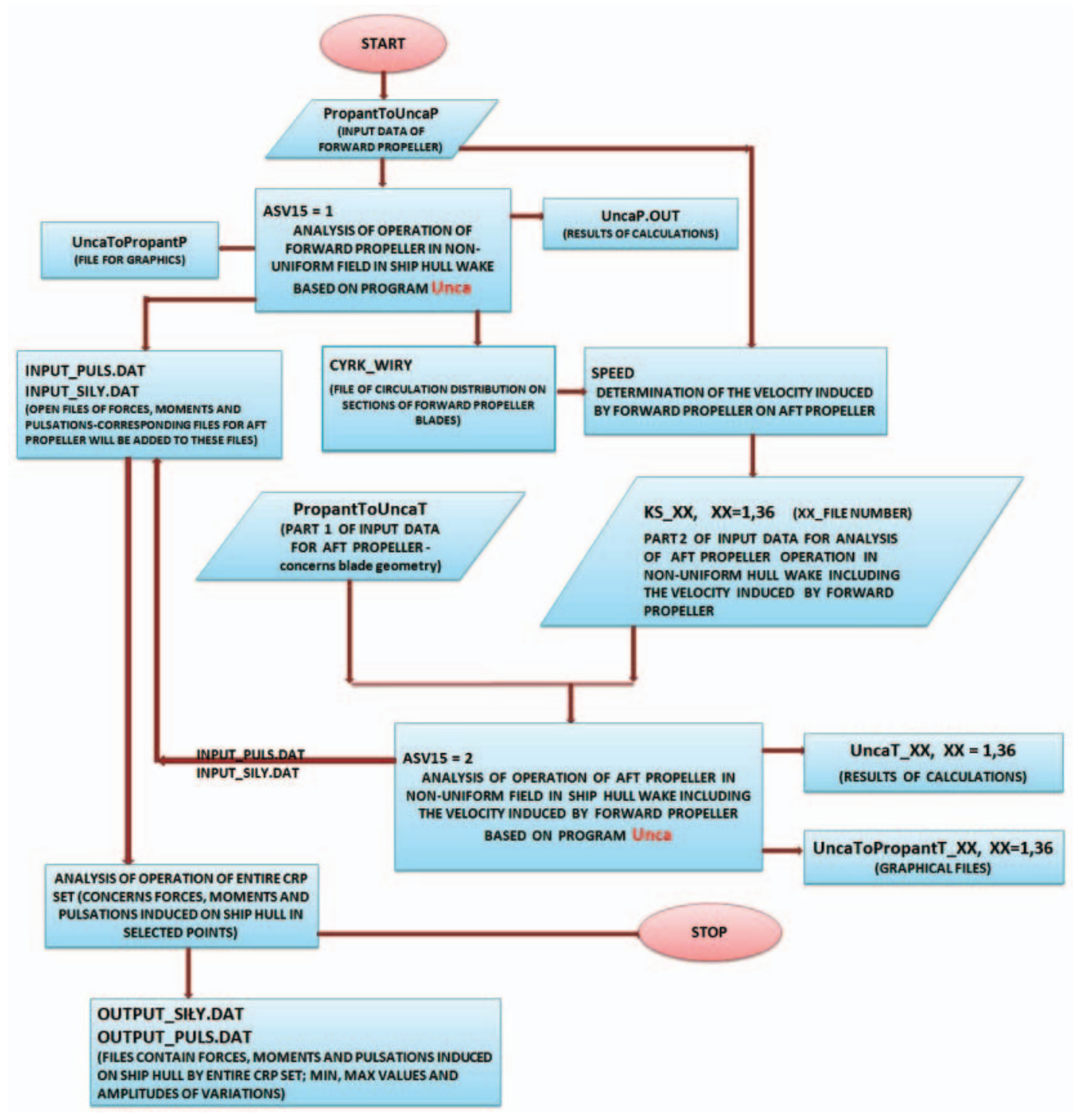

Fig. 3. The block diagram of the analysis procedure of the contra-rotating propellers

\section{PRESENTATION OF THE SELECTED BLOCKS OF THE NEW COMPUTER SYSTEM}

The computer system for design of the contra-rotating propellers has several blocks identical to those incorporated in the systems for design of single or tandem co-rotating propellers. Only the blocks concerning the specific problems of contra-rotating propellers are described in detail below.

\section{The input data}

The input data include all information required for initiation of the variant of design calculation selected out of four available options, similarly as for other types of propellers described in earlier publications $[4,5,6]$. The input data may be introduced either in the form of the previously prepared input data file or directly from the computer terminal in an interactive mode.

The input magnitude which is typical both for the tandem co-rotating and contra-rotating propellers is the axial distance between the generator lines of the forward and aft propellerThe features distinguishing the contra-rotating propellers from the tandem co-rotating propellers is the difference in diameters of the forward and aft propellers and the recommended difference in number of blades and rate of rotation. The diameter of the aft propeller must be smaller than the local diameter of the slipstream shed from the forward propellerThis requirement is based on two reasons: firstly there is a marked jump in velocity at the slipstream boundary (see Figs 9 and 10), which cannot be accommodated by the blade geometry (it would require a corresponding jump in the blade pitch) and secondly, there is danger of cavitation erosion caused by the tip vortices shed from the forward propeller.

The program enables graphical control and correction of the input data, especially the radial distributions of geometrial parameters such as blade outline, maximum thickness of the blade section profiles, blade skewback, inflow velocity in the ship wake etc. Fig. 4 shows an example of correction of the radial distribution of the circumferentially averaged inflow velocity in the ship wake.

\section{The design program}

The algorithm of the design program for contra-rotating propellers differs in the following details from the analogical programs for single or tandem co-rotating propellers $[4,6]$ :

a) the calculations are performed only for the given value of the total thrust of the CRP set

b) the division of the total thrust between the forward and aft propeller may be given in the input data or may be determined in the design program 


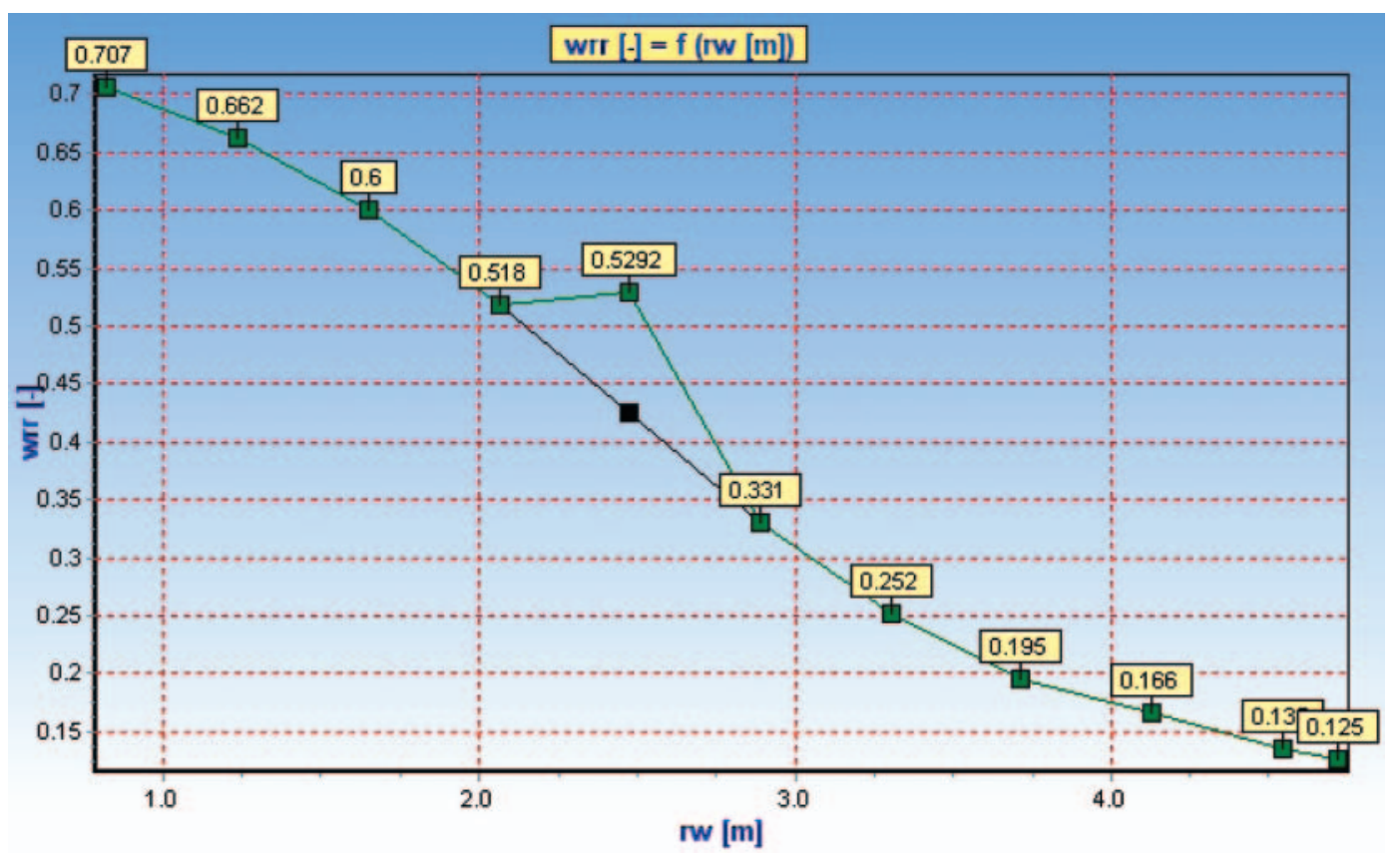

Fig. 4. The control display of the given radial distribution of the circumferentially averaged velocity in the ship wake, showing the correction of the erroneously set value

c) the diameter of the aft propeller may be given in the input data or may be determined in the design program (the determined value may still be corrected by the designer during calculations)

d) the determination of the velocity field induced by both propellers of the CRP set is necessary (this is done automatically in the design program).

The design calculations are performed in the same way for every selected version of the design task (similarly as in the case of classical single propellers).The results of design calculations are available in the form of the appropriate numerical files and drawings on the computer screen, which may be saved and, or printed. An example of such a drawing is shown in Fig. 5This drawing may be viewed on the screen from different angles and then printed in the most suitable configuration.

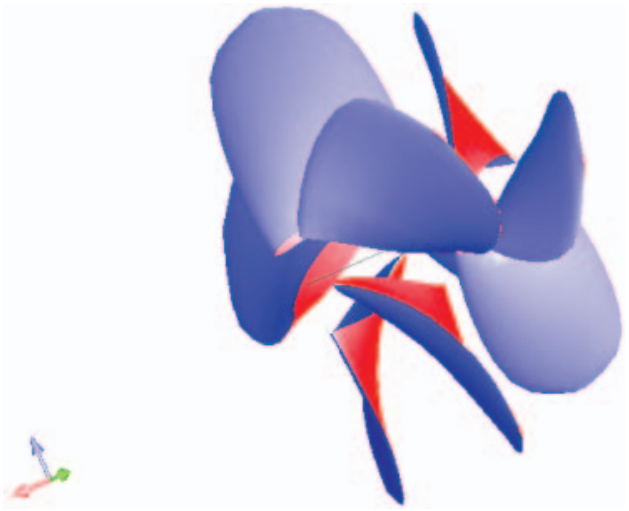

Fig. 5. The contra-rotating propellers as represented in the design program

As it has been mentioned above, the determination of the velocity fields induced by the bound and trailing vortex systems representing both propellers of the CRP set is an indispensable element of the design program. It may be seen in the block diagram shown in Fig. 2 that the design of CRPs an iterative process based on the mutual hydrodynamic interaction between the both propellers of the CRPset. The results of these calculations are presented in the format similar to that of the tandem co-rotating propellers [6].

\section{The induced velocity field}

In the propeller design program based on the vortex theory the systems of bound and trailing vortices are determined in a simplified form, which is sufficient for calculation of the velocities induced on the blades of the propellers, but it may be insufficient for calculation of the velocities induced at certain distance in front and behind the propeller blades (i.e. at locations of the respective propellers of the CRP or tandem co-rotating set of propellers).

The trailing vortex system of any propeller under goes contraction, deformation and concentration (rolling-up of vortices with simultaneous viscous dissipation of vorticity) $[7,8,9,10]$. As a result, tip vortices are formed behind every blade and a strong hub vortex is formed behind the propeller hub. The velocity field induced by such a system of concentrated vortices is strongly three-dimensional, being a function of all three co-ordinates (for example $\mathrm{x}, \Phi, \mathrm{r}$ in the cylindrical system). In the design task of CRP set we are interested in the circumferentially averaged values of the induced velocities, while in the case of tandem co-rotating propellers the fully local induced velocity field must be known. The examples of the induced velocity field behind a propeller in the selected cross-section $\mathrm{x}=$ const of the slipstream are shown in Figs 6, 7 and 8 . The velocities induced by the aft propeller at the location of the forward propeller have much smaller values and more uniform spatial distribution.

In case of the CRP set the velocity field induced by the forward propeller, combined with the inflow velocity field of the hull wake is an important design parameter. All three velocity components of that induced velocity field are averaged circumferentially (on the basis of the flow volumetric intensity) for a required number of radii. The radial distribution of these averaged velocity components shows a marked discontinuity at the local tip vortex radius.At the distances behind the propeller larger than the propeller radius both velocity components (axial and tangential) are equal zero outside the slipstream and they reach quite high values inside the slipstream. Such a sudden jump of the velocity cannot be accommodated by the appropriate geometry of the aft propeller blades. Hence the requirement to reduce the aft propeller diameter to the value 


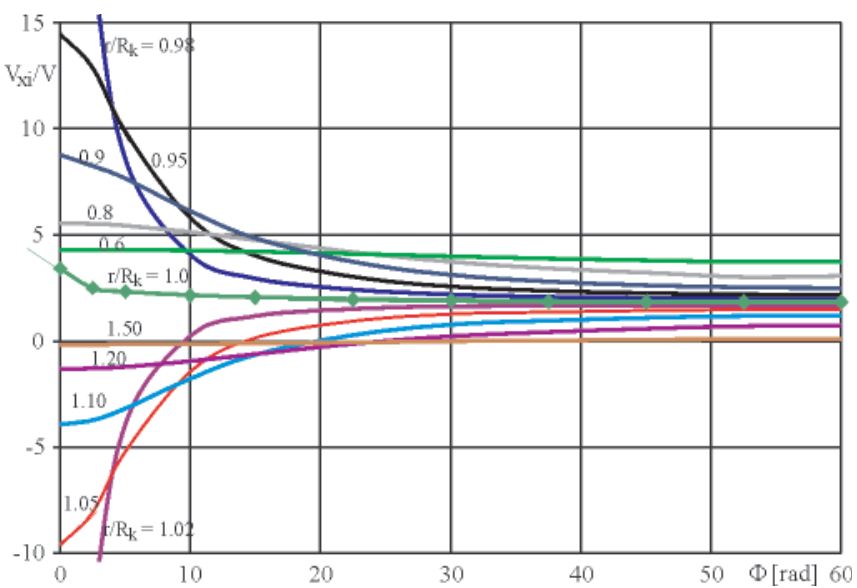

Fig. 6. The axial component of the velocity induced behind a three-bladed propeller

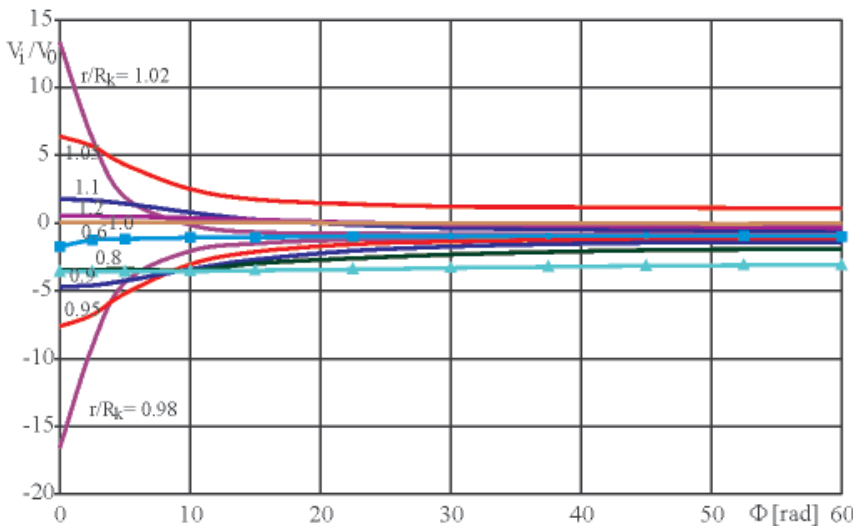

Fig. 7. The tangential component of the velocity induced behind a three-bladed propeller

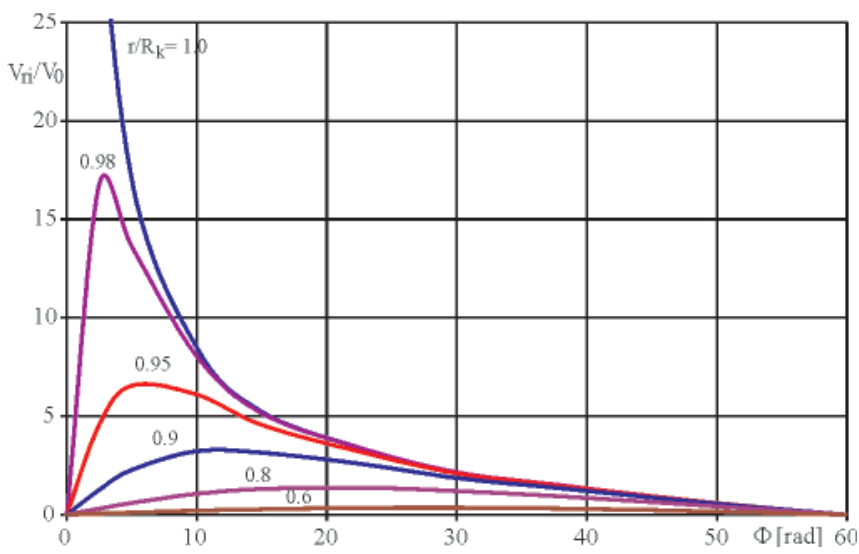

Fig. 8. The radial component of the velocity induced behind a three-bladed propeller

which ensures that the tips of the blades are already inside the slipstream shed from the forward propeller.

The volumetric averages of the velocities induced in front of the aft propeller are much smaller, their radial distribution is smooth and it does not exhibit any discontinuities (the volumetric average of the tangential induced velocity in front of the propeller is theoretically equal zero).

\section{The program for analysis of the contra-rotating propellers operation in the non-uniform velocity field}

The computer program UNCA for the analysis of the ship propeller operation in the non-uniform velocity field is a very important element of the design process of all propeller typesThe central part of the algorithm of this program is the determination of the size and extent of various forms of cavitation on the blades of the propeller operating in the circumferentially non-uniform velocity field. The original theoretical model combines the unsteady vortex lifting surface model with the model of unsteady sheet cavitation bubble. The detailed description of the algorithm of UNCA may be found in $[18,19]$.

In the case of a CRPset the modification of the non-uniform velocity field of the ship hull wake due to the velocity field induced by the propeller other than this currently analyzed is a very important part of the computation algorithm. In order to fulfil this task an additional procedure has been incorporated in the program This procedure calculates the induced velocity field both in front and behind the propeller.

The appropriately modified velocity field enables the detailed analysis of the operation of both forward and aft propeller by means of the program UNCA. In comparison with other propeller types the analysis of the CRP operation in the non-uniform inflow is particularly complicated and time-consuming. The aft propeller of the set operates in the non-uniform velocity field being the result of superposition of the hull wake and the velocity field induced by the forward propeller (see Figs. 9, 10 and 11). This analysis is additionally complicated by the fact that for each angular position of the forward propeller all selected angular positions of the aft propeller must be analyzedThe total number of the analyzed angular blade positions depends also on the number of blades of both propellers of the CRPset, but as a rule this number exceeds one thousand (for example for $\mathrm{Zf}=5$ and $\mathrm{Za}$ $=4$ the total number of the analyzed angular blade positions is $n=35 * 36=1260$ ). For each of these blade positions the appropriate numerical files containing results are generated, which under go further re-calculations, but they also may be presented on the computer screen in the numerical or graphical form or directly printed. These results include, among other data, the calculated cavitation phenomena on the aft propeller, shown in Fig. 12.

The results of calculations of the unsteady hydrodynamic forces and moments on the aft propeller for the angular position of the forward propeller equal toAF $=0$ are presented below (Figs. 13, 14 and 15). Similar diagrams may be presented for every mutual position of the forward and aft propeller. Independently, analogical results are available for the forward propeller.

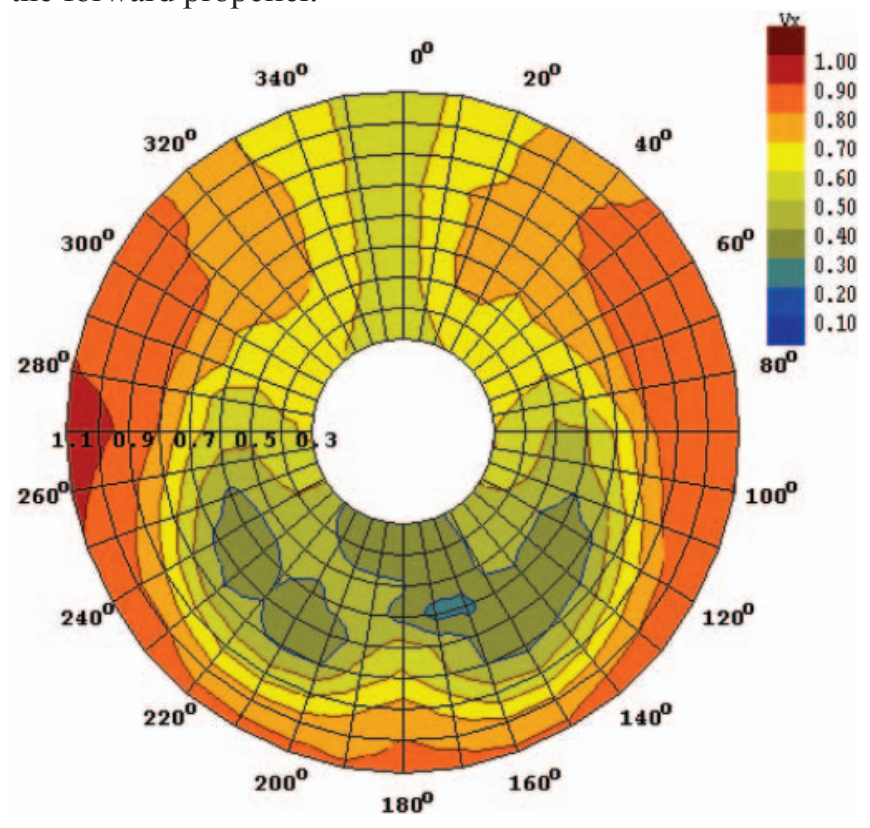

Fig. 9. The velocity field behind the ship hull 


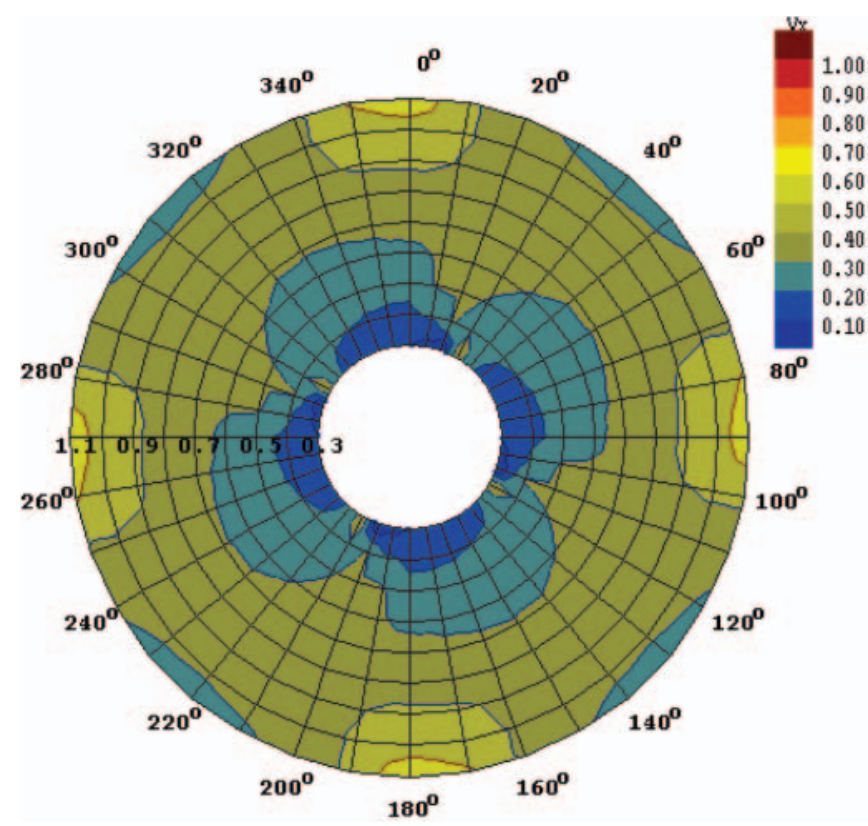

Fig. 10. The velocity induced by the forward propeller at the location of the aft propeller at the angular position of the forward propeller $A F=0$

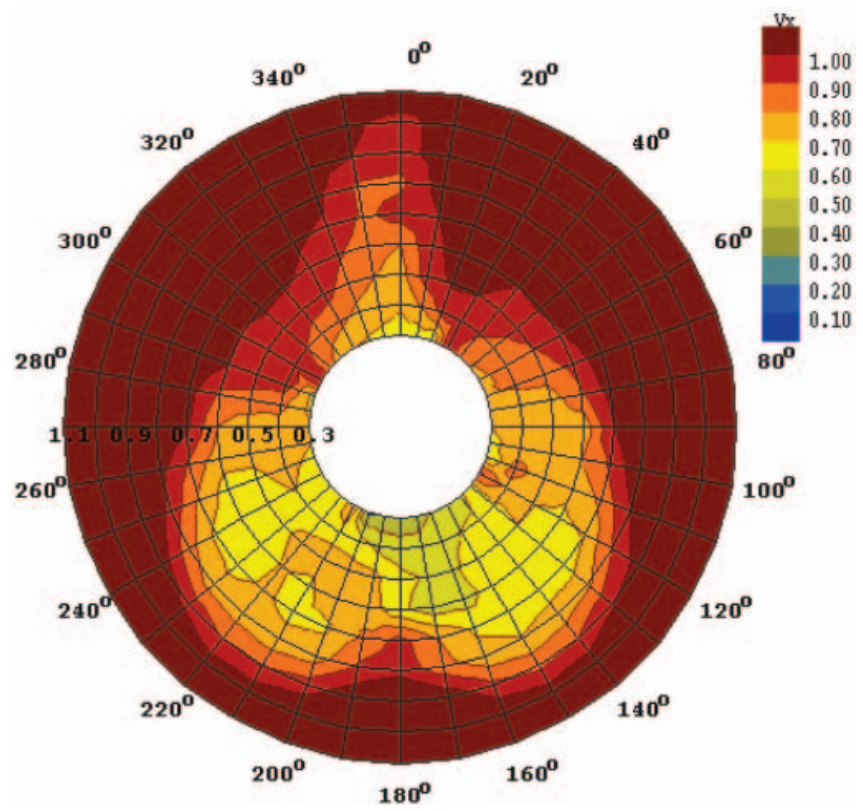

Fig. 11. The resultant velocity field at the location of the aft propeller for the angular position of the forward propeller $A F=0$
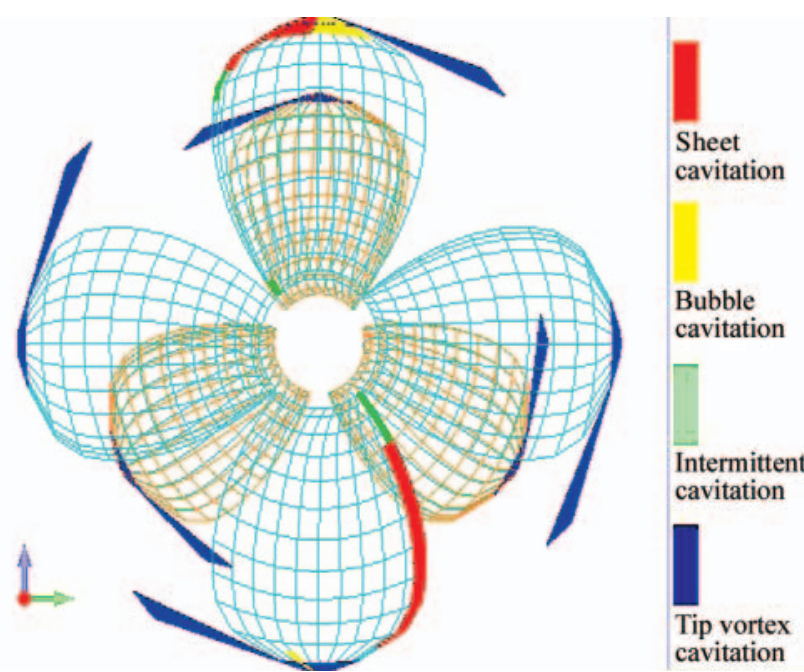

Fig. 12. The example of computed cavitation phenomena on the contra-rotating propellers

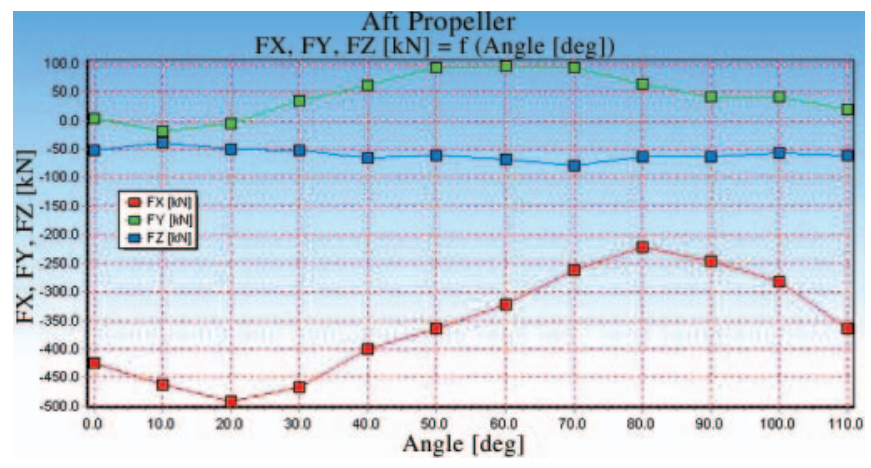

Fig. 13. The computed hydrodynamic force components $F X, F Y, F Z$ on the aft propeller for the angular position of the forward propeller $A F=0$

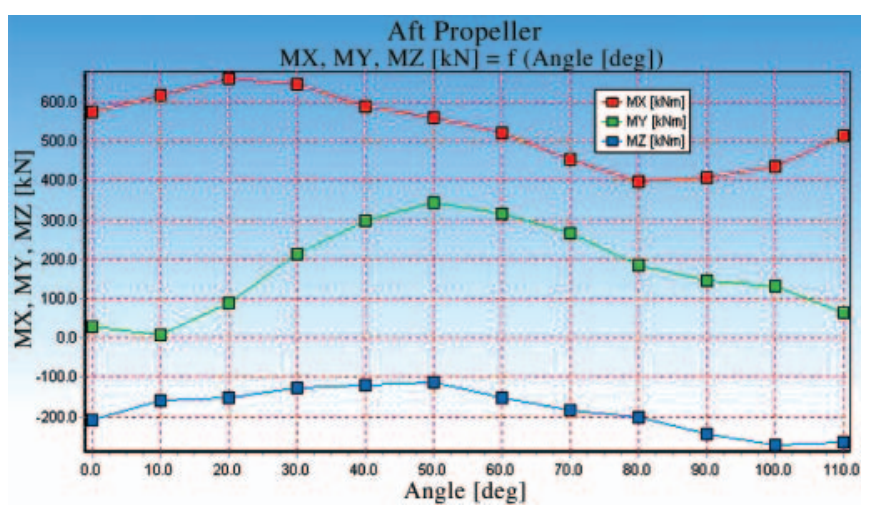

Fig. 14. The computed hydrodynamic moment components $M X, M Y, M Z$ on the aft propeller for the angular position of the forward propeller $A F=0$

For every angular blade position of the forward propeller of CRP the maximum and minimum values of the calculated pressure pulsations generated on the hull by the aft propeller and of the unsteady shaft forces are extracted. These data are presented in the numerical and graphical form (see Figs. 17 and 21).

Both propellers of the CRP set are analyzed by means of the program UNCA from the following points of view:

a) the intensity and extent of the different forms of cavitation in different angular positions of both propellers in the combined non-uniform velocity field including the hull wake and the propeller-induced velocities,

b) the level of pressure pulsations generated by the CRP set and by the forward and aft propeller separately on the hull surface or in the surrounding space,

c) the level of the fluctuating hydrodynamic shaft forces and moments for the forward and aft propeller separately.

After such an analysis the designer may return to the CRP design calculation, modifying the selected details of the geometry and hydrodynamic parameters of both propellers of the set:

a) the radial distribution of blade skewback

b) the radial distribution of the blade section chord lengths

c) the radial distribution of the maximum blade section thickness

d) the type of blade section thickness distribution

e) the type of blade section mean line

f) the radial distribution of hydrodynamic loading of the blades

g) the number of blades of both propellers

h) the division of CRP total thrust between the forward and aft propeller

i) the diameter of one or both propellers of CRP. 
The above described analysis of CRP is performed only for the design condition, because any change of the ship speed or the propellers rates of rotation changes the operating conditions of both propellers - consequently the fields of induced velocities of both propellers change in the qualitative and quantitative sense.

The numerical results of the above analysis are stored in three separate files, referring respectively to the forward propeller, aft propeller and to the entire CRP set.

The method of graphical presentation of the results is similar to that applied to the classical single propellers [4], with the modification that in case of CRP it may refer separately to the forward or aft propeller or to the entire CRPset. The examples of such presentation are shown in the next section.

\section{COMPARATIVE ANALYSIS OF CRP AND TANDEM CO-ROTATING PROPELLERS}

In the preceding publications $[4,6]$ the results of design calculations of the single propeller and of the tandem corotating propellers. The case of the same lage fast cargo vessel has been used in the design of the CRBet described below. The design ship speed is $\mathrm{V}=25.3$ [knots] and the required thrust of the propulsor is $\mathrm{T}=3250[\mathrm{kN}]$ The design rate of rotation of the engine and of the forward propeller is 100 [rpm]. In the case of CRP set the rate of rotation of the aft propeller may be different and it may be optimized from the point of view of propulsive efficiency, cavitation, pressure pulsations on the hull and unsteady hydrodynamic shaft forces. In the process of such an optimization the best performance of CRP was achieved for the number of blades of the forward propeller $\mathrm{Zf}=5$, number of blades of the aft propeller $\mathrm{Za}=3$ and for the rate of rotation of the aft propeller equal to 115 [rpm]. The optimum diameter of the aft propeller was determined on the basis of the appropriate semi-empirical formulae concerning the slipstream behind the forward propeller . The calculated main characteristics of the forward and aft propellers of the CRP set are given in Tab. 1 below.

Tab. 1. Main parameters of the designed CRP set

\begin{tabular}{|c|c|c|}
\hline Characteristic & $\begin{array}{c}\text { Forward } \\
\text { propeller }\end{array}$ & Aft propeller \\
\hline Propeller diameter [m] & 7.80 & 5.83 \\
\hline Expanded blade area ratio [-] & 0.59 & 0.42 \\
\hline Shaft power [kW] & 30060 & 11800 \\
\hline Mass of the blades [kg] & 23343 & 7300 \\
\hline $\begin{array}{c}\text { Static moment of inertia } \\
{\left[\mathrm{kgm}^{*} \text { 2] }\right.}\end{array}$ & 511944 & 76419 \\
\hline
\end{tabular}

The comparison of the above results for CRP with those obtained for other propeller types $[4,6]$ shows that the propulsive efficiency of the CRPset is about 5 percentage points higher than that of a single propeller and about 2.5 percentage points higher than the tandem co-rotating propellers. However the CRP set is worse than the other analyzed propellers as far as the pressure pulsations on the hull (probably also acoustic pressures) and the unsteady hydrodynamic shaft forces are concerned.

Figs. 15 and 16 below present the calculation results for the CRP set, concerning the harmonic amplitudes of the unsteady hydrodynamic shaft forces and moments, shown separately for the forward and aft propeller Presentation of the results is done separately for each of the propellers of the CRP set because their different numbers of blades and diferent rates of rotation prohibit presentation of the results on one common diagram of harmonic amplitudes.
Moreover, the calculations of the harmonic amplitudes of the unsteady hydrodynamic shaft forces are performed separately for each angular position of the forward and aft propellers. The numerical files containing the results or their graphical representations may be inspected. However, the analysis of about 1000 diagrams or files is not easy. In order to facilitate this analysis, for each angular position of one of the propellers only the maximum and minimum values of the respective components of the unsteady shaft forces and moments are extracted. These values are appropriately added together and presented in the form of a relatively short numerical file or as one diagram. Example of such a diagram is presented in Fig. 17. This diagram includes also the diferences between the maximum and minimum values because these differences are decisive parameters in some Classification Society Rules.

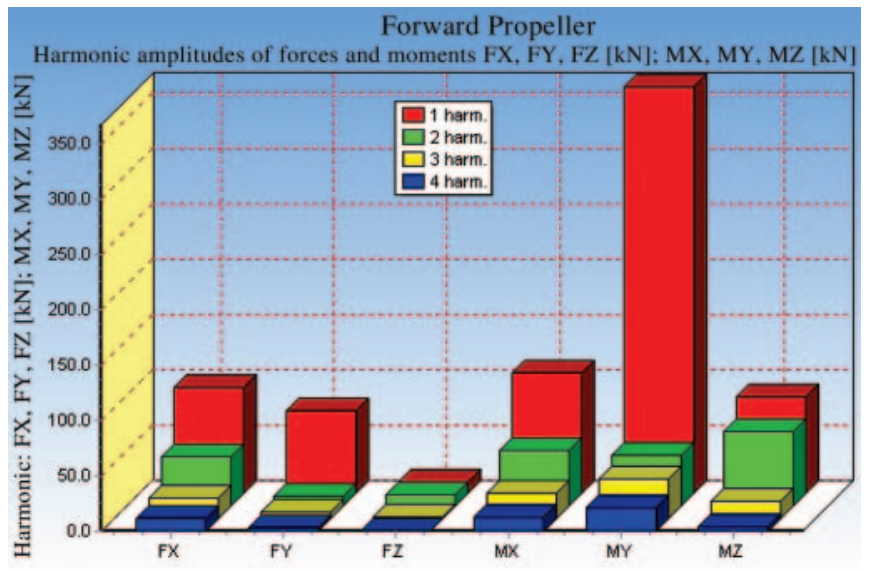

Fig. 15. The computed harmonic amplitudes of the hydrodynamic force and moment components for the forward propeller of the CRP set

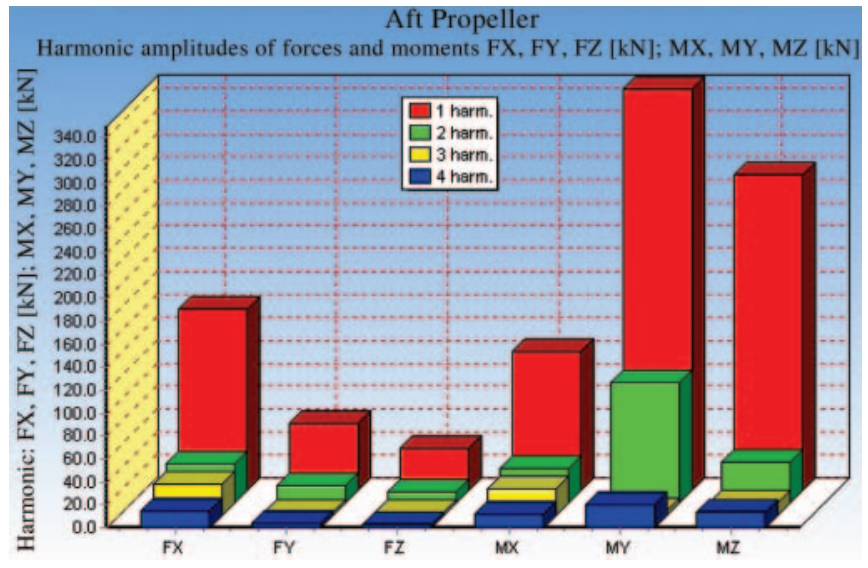

Fig. 16. The computed harmonic amplitudes of the hydrodynamic force and moment components for the aft propeller of the CRP set for the angular position $A F=0$ of the forward propeller

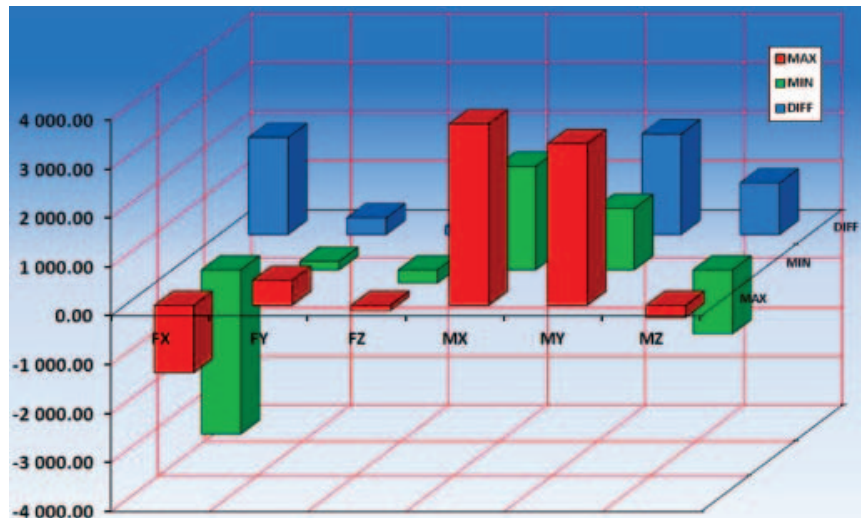

Fig. 17. Maximum and minimum values, together with their differences, for the hydrodynamic shaft forces [kN] and moments [kNm] on the entire CRP set 
For the sake of comparison the corresponding results of calculations for the tandem co-rotating set, designed for the same ship and operating in the same non-uniform velocity field [6], are presented in the analogical format in Fig. 18.

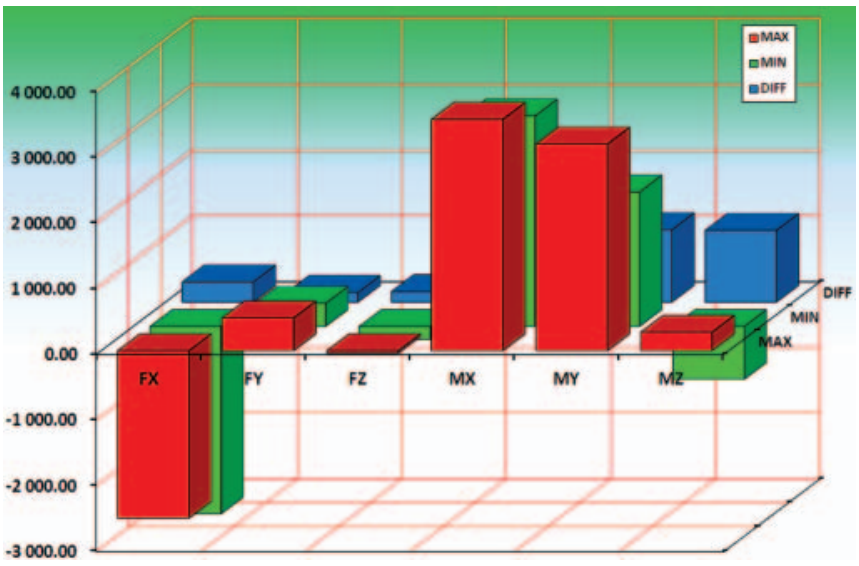

Fig. 18. Maximum and minimum values, together with their differences, for the hydrodynamic shaft forces [kN] and moments [kNm] on the set of tandem co-rotating propellers

A similar presentation of the calculation results may be done for the pressure pulsations generated on the hull (or acoustic pressures in the surrounding space). In Figs. 19 and 20 the harmonic amplitudes of the pressure pulsations generated in the selected points on the hull by each propeller of the CRP set separately are presented. Fig. 21 shows the maximum and minimum values of these pressure pulsations, together with their dif ferences. For the sake of comparison, the corresponding values calculated for the tandem co-rotating propellers, designed for the same ship and operating in the same non-uniform velocity field are shown in Fig. 22.

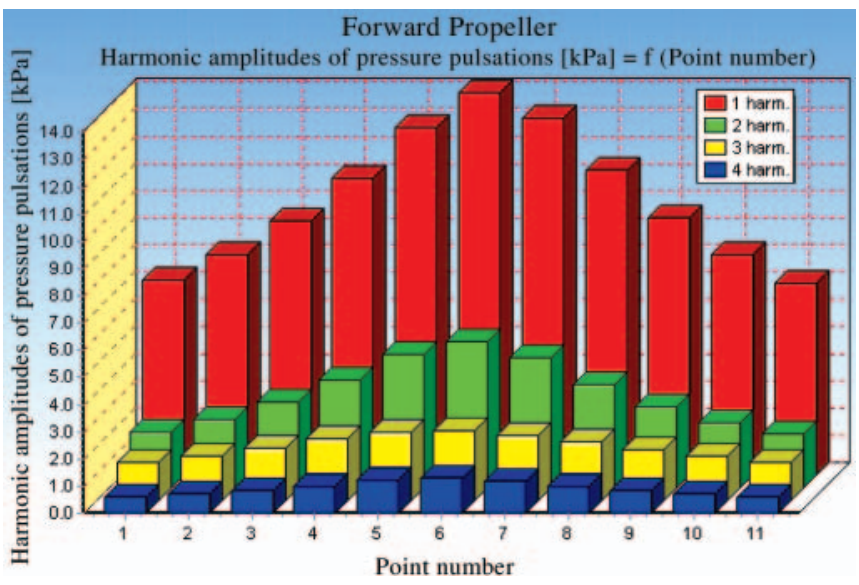

Fig. 19. Harmonic amplitudes of the pressure pulsations generated on the hull by the forward propeller of the CRP set

Similarly as in the case of other propeller types, the program determines the presence and extent of the various cavitation forms, which are described as numerical files or corresponding illustrations. In Figs. 23, 24 and 25 the examples of graphical presentation of the computed cavitation phenomena are shown.

The cavitation pictures may be viewed independently for each of the propellers (Figs. 23 and 24) or in combination (Fig. 25). Apart from the static pictures the program enables viewing the CRP set in motion, exhibiting the calculated unsteady cavitation phenomena. The complete revolution of both propellers of the CRP set may be presented, showing the time-dependent variation of the cavitation phenomena on the propeller blades in the non-uniform velocity field (in this presentation the forward or aft propeller is alternatively immobilized and the other performs one full revolution). This presentation may be also recorded in the format of a film.

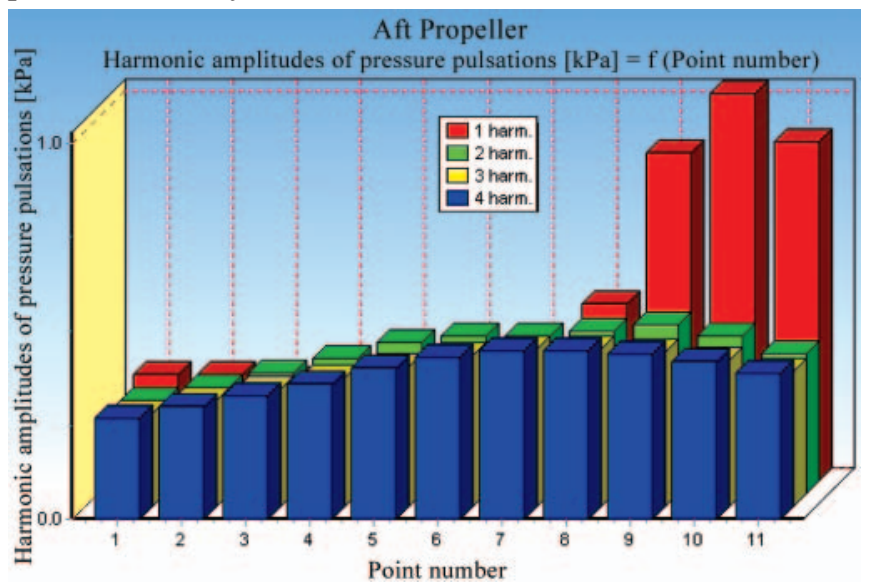

Fig. 20. Harmonic amplitudes of the pressure pulsations generated on the hull by the aft propeller of the CRP set

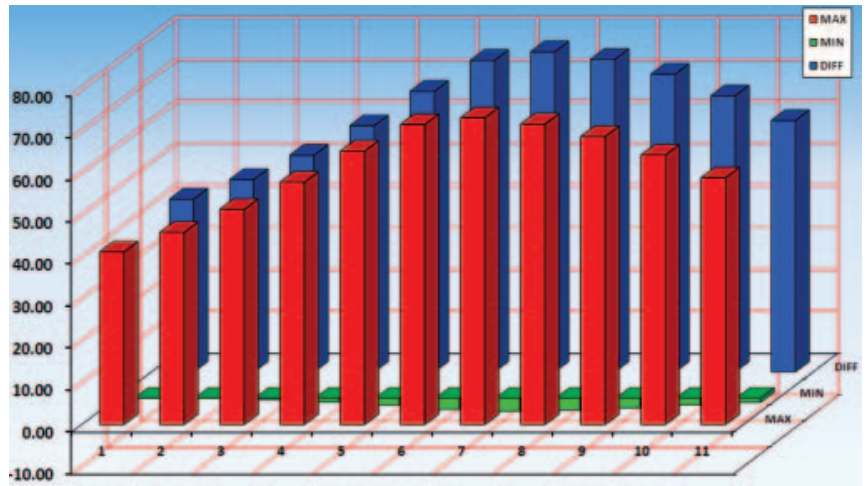

Fig. 21. Maximum and minimum values, together with their differences, of the pressure pulsations $[\mathrm{kPa}]$ induced on the hull by the entire CRP set

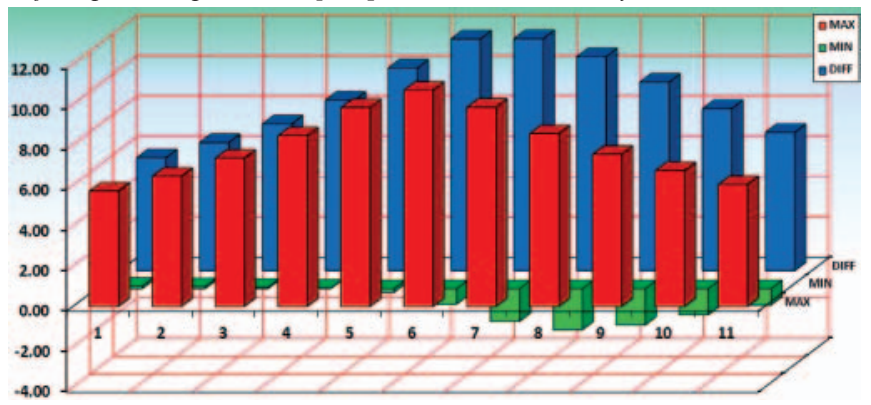

Fig. 22. Maximum and minimum values, together with their differences, of the pressure pulsations [kPa] induced on the hull by the entire set of tandem co-rotating propellers

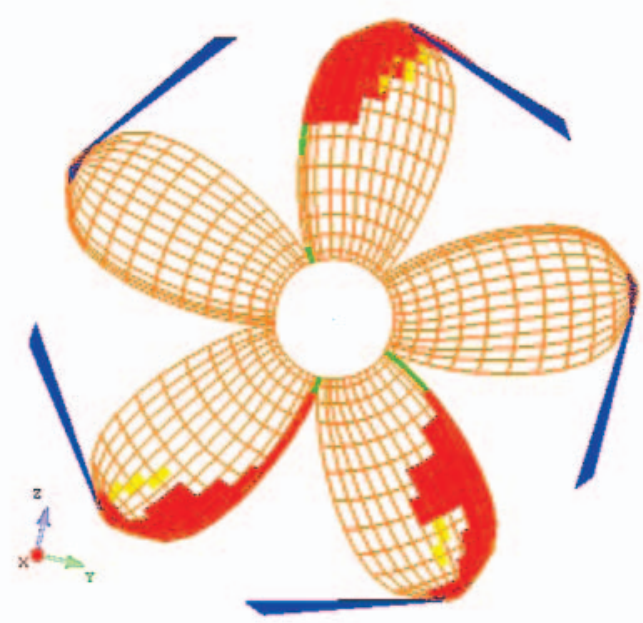

Sheet cavitation

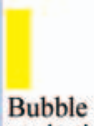
cavitation

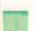

Intermittent cavitation

Fig. 23. Example of the computed cavitation phenomena on the forward propeller of the CRP set 

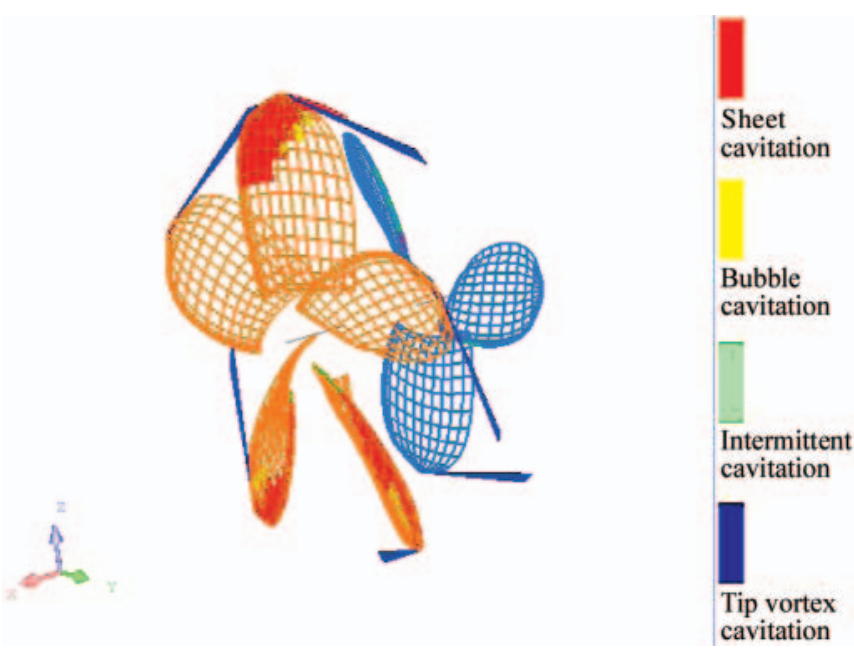

Fig. 24. Example of the computed cavitation phenomena on the aft propeller of the CRP set
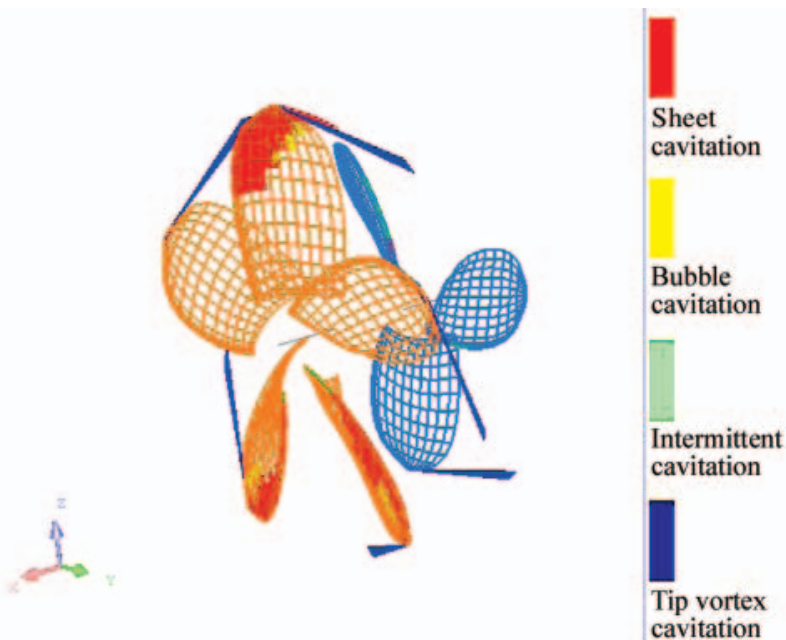

Fig. 25. Example of the computed cavitation phenomena on both propellers of the CRP set for their selected position in the non-uniform inflow

It may be concluded from the analysis of the above examples, that from the point of view of propulsive efficiency the CRP set is better than the tandem co-rotating propellers and than the classical single propeller This is not a general rule, the design example presented in $[4,5,6]$ is selected in such a way, that it may be demonstrated that in certain areas of propeller operation the CRP or tandem co-rotating propellers may be more efficient than the classical single propeller.

The problem of cavitation performance, unsteady hydrodynamic shaft forces and pressure pulsations is much more involved and it requires a thorough analysis of the results of comparative calculations. The computer systems presented in the current line of publications $[4,5,6]$ facilitate the optimum selection of the appropriate propeller type for every application.

\section{CONCLUSIONS}

- The computer system presented above fully facilitates the process of design of the contra-rotating propellers. This system incorporates all elements necessary in the correct design process of CRP. The computational process itself is relatively fast (although much slower than in the case of other, simpler propellers), and the graphical subroutines enable efficient analysis of the consecutively designed CRP sets, directing the whole process towards an optimum solution of the design task.
- The relatively short computation time allows for calculation of a large number of variants of the designed CRP set. For example the following parameters may be modified in the course of design without leaving the system:

- number and geometry of the blades of both propellers of the CRP set (including the geometry of the blade sections)

- diameters of both propellers of the CRP set

- ship speed and rates of rotation of both propellers of the CRP set (equal or different)

- division of the total thrust between the forward and aft propeller of the CRP set

- radial distribution of the hydrodynamic loading of the blades

- axial distance between both propellers of the CRP set.

- The above listed modifications enable the optimization of the CRP set from the point of view of efficiencycavitation performance, induced pressure pulsations and unsteady hydrodynamic shaft forces and moments. The widely employed graphical presentation of the results greatly facilitates such an optimization analysis.

- The above presented design example, together with the results of calculations of the ducted propeller included in [5] and tandem co-rotating propellers included in [6], lead to the conclusion that it is worthwhile to include in the design analysis different propeller types. The four propeller design systems presented in the current line of publications $[4,5,6]$, which accept very similar input data files (relatively the most onerous part of the design process), enable very fast and efficient comparative analysis, leading to the selection and consecutive design of the optimum propeller type in every practical application.

\section{BIBLIOGRAPHY}

1. Bjaerne E.: Systematic Studies of Contra-rotating Propellers for Merchant Ships, Transactions RINA 1973

2. Glover E.J.: Contra-rotating Propellers for High Speed Cargo Vessels, Transactions NECIES, February 1967

3. van Gunsteren L.A.: Application of Momentum Theory in Contra-rotating Propellers, International Shipbuilding Progress, No. 206, 1971

4. Koronowicz T., Krzemianowski Z., Tuszkowska T., Szantyr J.A.: A Complete Design of Ship Propellers Using the New Computer System, Polish Maritime Research No. 1, vol. 16, 2009

5. Koronowicz T., Krzemianowski Z., Tuszkowska T., Szantyr J.A.: A Complete Design of Ducted Propellers Using the New Computer System, Polish Maritime Research No. 2, vol. 16, 2009

6. Koronowicz T., Krzemianowski Z., Tuszkowska T., Szantyr J.A.: A Complete Design of Tandem Co-rotating Propellers Using the New Computer System, Polish Maritime Research No. 4, vol.16, 2009

7. Koronowicz T.: Algorithm of the Design Program Based on the Single Layer Lifting Surface Model Applied to the Arbitrarily Skewed Propellers (in Polish), IFFM Report No. 295/1986

8. Koronowicz T.: Analysis of the Velocity Field of the Helical Vortices (in Polish), IFFM Report No. 643/1969

9. Koronowicz T.: Determination of the Velocity Field in the Close Vicinity of the Screw Propeller (in Polish), IFFM Report No. 58/729/1973

10. KoronowiczT.: Determination of the Variation of Induced Velocities in the Propeller Wake (in Polish), IFFM Report No. 83/745/1974

11. LerbsH.W.: Contra-rotating Optimum Propellers Operating in Radially Non-uniform Wake, DTMB Report 941-1955 
12. LindgrenH.: Hydrodynamic Aspects of Contra-rotating Propellers, Shipping World and Shipbuilder, November 1967

13.van Manen J.D., Sentic A.: Contra-rotating Propellers, NSMB Publication No. 126/1956

14. MinsaasK.: A Method for the Design of Contra-rotating Propellers, Publication NSET No. 112, 1971

15. MłynarczykJ.: Design of the Contra-rotating Propellers on the Basis of Lerbs Theory (in Polish) IFFM Report No. 37/898/1978

16. Mogan W.B.: The Design of Contra-rotating Propellers Using Lerbs Theory, Transactions SNAME, 1960

17. SzantyrJ.A.: A Computer Program for Calculation of Cavittaion Extent and Excitation Forces for a Propeller Operating in the Non-uniform Velocity Field, International Shipbuilding Progress No. 296, vol. 26, 1979

18. SzantyrJ.A.: The Method of Deformable Lifting Surface Theory for Determination of the Unsteady Cavitation and its Hydrodynamic Consequences, Doctor of Science Thesis, IFFM Report No. 183/1110/1984

19. SzantyrJ.A.: A Method for Analysis of Cavitating Marine Propellers in Non-uniform Flow, International Shipbuilding Progress No. 427, vol.41, 1994

\section{CONTACT WITH THE AUTHORS}

Tadeusz Koronowicz, Prof. Zbigniew Krzemianowski, Ph. D. Teresa Tuszkowska, Ph. D.

Institute of Fluid-Flow Machinery, Polish Academy of Sciences Fiszera 14 80-952 Gdansk, POLAND e-mail: ttk@interecho.com

Jan A. Szantyr, Prof.

Department of Turbomachinery and Fluid Mechanics,

Gdańsk University of Technology Narutowicza 11/12

80-233 Gdansk, POLAND e-mail: jas@pg.gda.pl 